\title{
Filling of hole arrays with InAs quantum dots
}

\author{
Jennifer Y Lee ${ }^{1}$, Mark J Noordhoek ${ }^{1}$, Peter Smereka ${ }^{2}$, \\ Hugh McKay ${ }^{1}$ and Joanna M Millunchick ${ }^{1}$ \\ ${ }^{1}$ Department of Materials Science and Engineering, University of Michigan, Ann Arbor, \\ MI 48109, USA \\ ${ }^{2}$ Department of Mathematics, University of Michigan and the Michigan Center for \\ Theoretical Physics, Ann Arbor, MI 48109, USA
}

Received 16 April 2009, in final form 21 May 2009

Published 23 June 2009

Online at stacks.iop.org/Nano/20/285305

\begin{abstract}
Focused ion beams are used to pattern $\mathrm{GaAs}(001)$ surfaces with an array of nanometer-deep holes upon which deposition of InAs results in quantum dot formation at the hole location. Experiments show that the size and quantity of quantum dots formed depend on growth parameters, and ion dose, which affects the size and shape of the resulting holes. Quantum dots fabricated in this fashion have a photoluminescence peak at $1.28 \mathrm{eV}$ at $77 \mathrm{~K}$, indicating that the ion irradiation due to patterning does not destroy their optical activity. Kinetic Monte Carlo simulations that include elastic relaxation qualitatively model the growth of dots in nanometer-deep holes, and demonstrate that growth temperature, depth of the holes, and the angle of the hole sidewalls strongly influence the number of quantum dots that form at their perimeter.
\end{abstract}

(Some figures in this article are in colour only in the electronic version)

\section{Introduction}

The alignment of InAs quantum dots into regular and dense arrays has been vigorously pursued for applications in optoelectronics [1,2] and cryptography [3, 4]. A variety of schemes to control the nucleation locations of the dots have been examined, ranging from standard lithographic techniques [5-7] to atomic force lithography [8]. In situ focused ion beam (FIB) patterning has the capability of creating patterns on the order of a few hundred nanometers or less, without the need for ex situ processing [9-11]. As such, FIB patterning has emerged as a viable tool for the fabrication of photonic and optoelectronic devices $[12,13]$. In order to make use of these patterns in device applications, the resulting quantum dot arrays must be regular and uniform and retain their optical activity. In this paper, we examine the effect of the FIB patterning of GaAs substrates on the assembly of InAs quantum dots and show that they luminesce. Experiments show that the regularity of the resulting quantum dot arrays is most strongly dictated by the fidelity of the initial pattern of holes. A unique kinetic Monte Carlo simulation of the growth that includes elastic relaxation of the lattice shows that the location, size, and number of dots can be delicately tuned by controlling the growth conditions and the geometry of patterned hole.

\section{Experimental details}

In vacuo FIB patterning was used to direct the self-assembly of quantum dot arrays. Samples were grown using a molecular beam epitaxy system on GaAs(001) substrates. Typical values for growth rates were 0.75 monolayers $\mathrm{s}^{-1}\left(\mathrm{ML} \mathrm{s}^{-1}\right)$ for $\mathrm{Ga}$, and $0.25 \mathrm{ML} \mathrm{s}^{-1}$ for In. Two different As 4 fluxes, $\sim 1.0$ and $\sim 2.2 \mathrm{ML} \mathrm{s}^{-1}$, were employed to give similar III/V flux ratios while growing GaAs or InAs. All temperature measurements were made by an optical pyrometer. After oxide desorption, a $400 \mathrm{~nm} \mathrm{GaAs}$ buffer layer was grown under the higher $\mathrm{As}_{4}$ flux at a substrate temperature of $T=580^{\circ} \mathrm{C}$. The sample was then annealed at the growth temperature under a high $\mathrm{As}_{4}$ flux and allowed to cool to room temperature under low arsenic flux. When cool, the sample was transferred in vacuo to the FIB patterning system. The FIB was employed to pattern the GaAs substrate with arrays of holes with 200$500 \mathrm{~nm}$ spacing. Each hole was dosed in a single pass with a $30 \mathrm{keV}, 7.5 \mathrm{pA}$ beam using ion dwell times ranging from $400 \mu \mathrm{s} \leqslant t \leqslant 1600 \mu \mathrm{s}$, which have predicted sputter depths on the order of a few monolayers or less [14]. After irradiation, samples were returned to the growth chamber and the sample temperature was raised to $T=530^{\circ} \mathrm{C}$ under low $\mathrm{As}_{4}$ flux. Because the sample never left the vacuum system, 


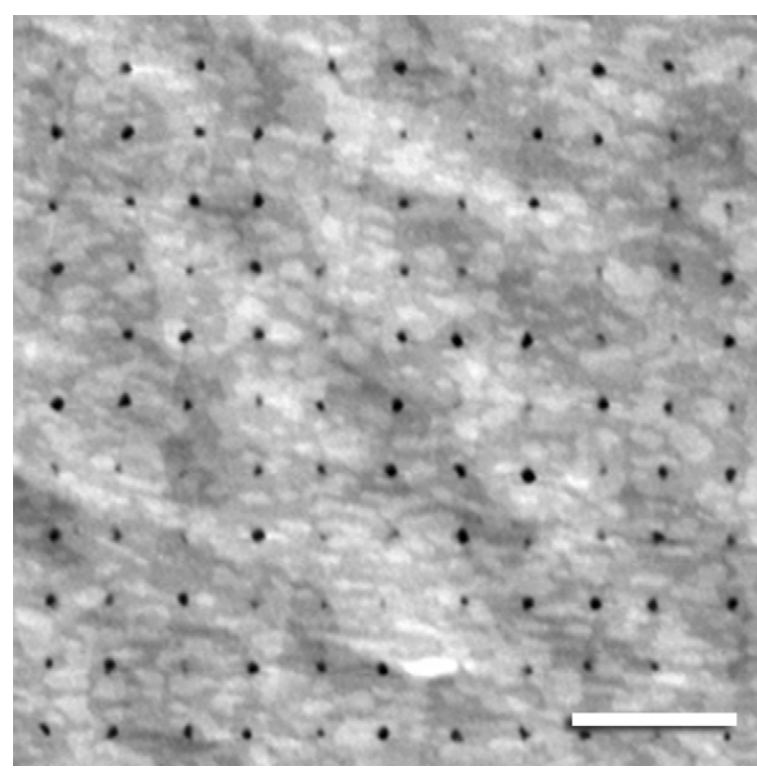

Figure 1. AFM images of a GaAs buffer layer that has been patterned with an array of holes spaced $250 \mathrm{~nm}$ apart and dosed with a dwell time of $800 \mu$ s per hole, followed by annealing at $T=530^{\circ} \mathrm{C}$ under low $\mathrm{As}_{4}$ flux. The scale bar is $500 \mathrm{~nm}$, and the height scale in $3 \mathrm{~nm}$.

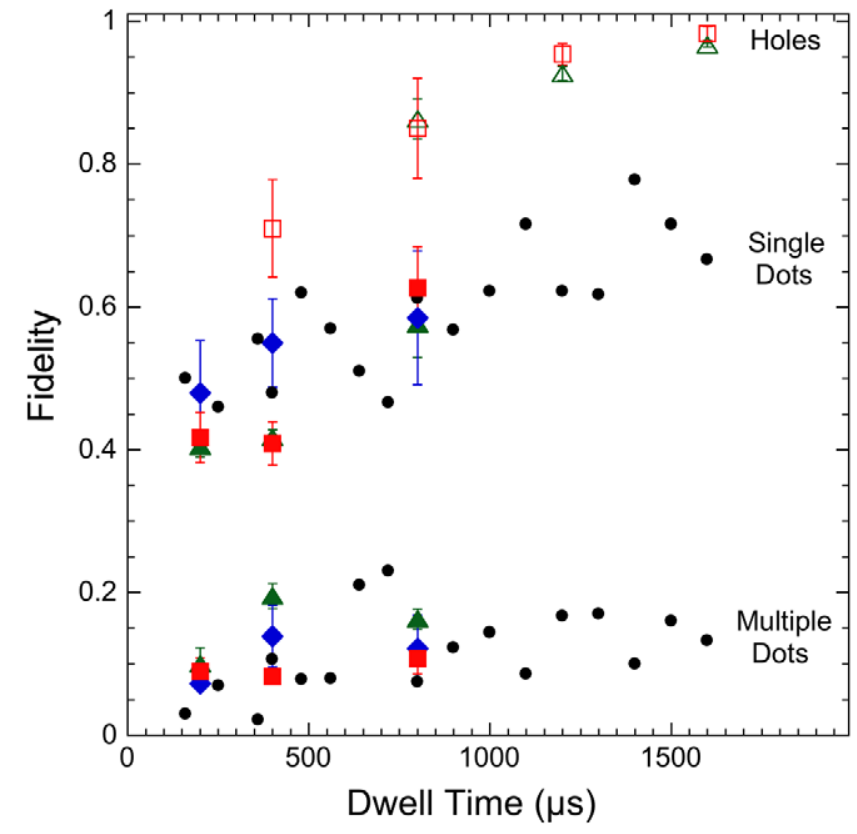

Figure 2. Probability of finding a hole, single quantum dot, or multiple quantum dots appearing at the intended location for a 2.0 ML-thick InAs film deposited at $T=530^{\circ} \mathrm{C}$ for arrays with periodicity of $140 \mathrm{~nm}$ (green triangle), $275 \mathrm{~nm}$ (red square), $500 \mathrm{~nm}$ (black circle), and $550 \mathrm{~nm}$ (blue diamond).

issues often observed with regard to preferential oxidation of ion-irradiated areas $[15,16]$ are circumvented. Thin layers of InAs $(1.7 \mathrm{ML}<h<2.4 \mathrm{ML})$ were deposited at $T=510$ or $530^{\circ} \mathrm{C}$ under a low arsenic flux while the surface had a $c(4 \times 4)$ reconstruction according to reflection high energy electron diffraction. Following growth, samples were quenched to
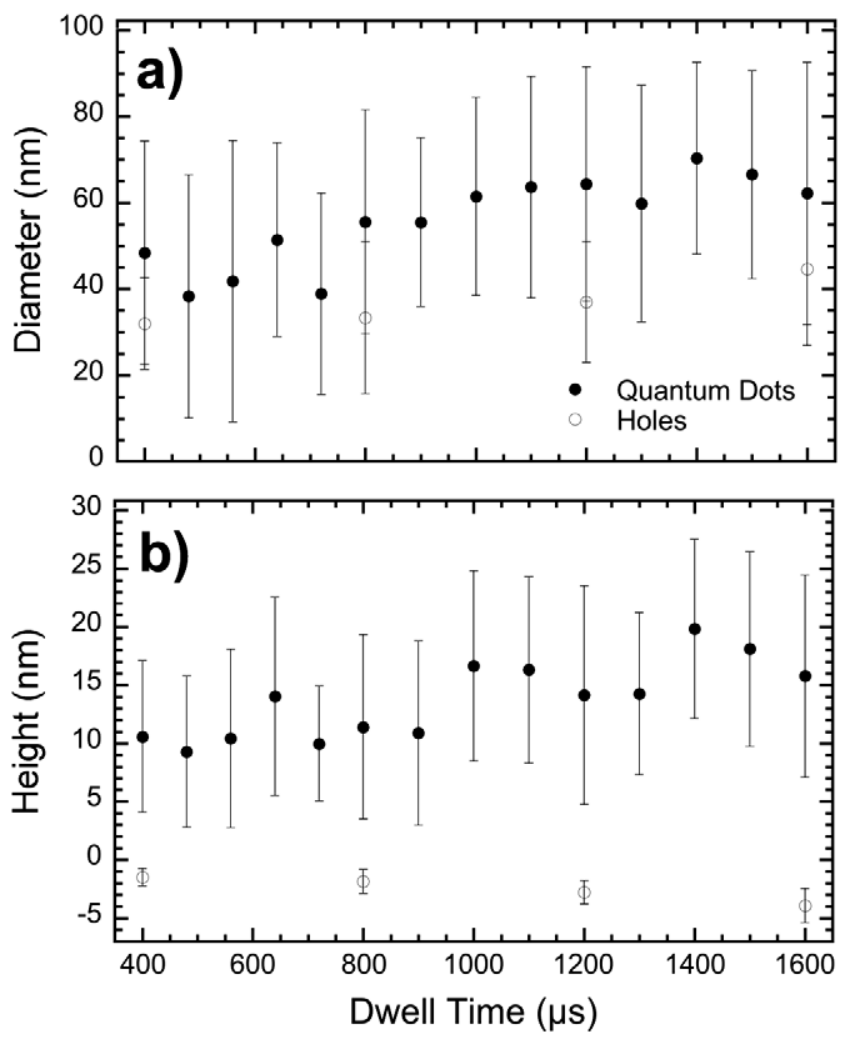

Figure 3. Plot of the feature (a) diameter and (b) height as a function of dwell time for holes (open circles) and quantum dots (closed circles).

room temperature under low $\mathrm{As}_{4}$ flux. The samples were characterized ex situ using an atomic force microscope (AFM) in tapping mode, using tips with a radius of curvature of $8 \mathrm{~nm}$.

\section{Results and discussion}

Figure 1 shows an AFM image of a GaAs buffer layer that has been patterned with an array of holes spaced $250 \mathrm{~nm}$ apart and dosed with a dwell time of $800 \mu$ s per hole followed by an anneal to $T=530^{\circ} \mathrm{C}$ under low $\mathrm{As}_{4}$ flux. For this set of patterning conditions, individual holes due to ion irradiation are clearly observed that are $42 \pm 13 \mathrm{~nm}$ in diameter and $1.7 \pm 0.7 \mathrm{~nm}$ deep. The step structure of the substrate is also resolved between the holes and is apparently unperturbed by the patterning process. The hole size varies, and in some instances no hole is visible at the expected location. The probability of finding a hole at the intended location, which we call the fidelity, increases as a function of ion dose (plotted in figure 2) and approaches 1 for the highest dwell times $(1600 \mu \mathrm{s})$. The periodicity of the hole array does not affect the probability of finding a dot at the intended location for this set of irradiation conditions. The fact that at lower dwell times the fidelity is not unity suggests that the hole formation process has a stochastic component. Indeed, it has been shown that the $\mathrm{Ga}^{+}$ion beam initially injects point defects into the surface, which then coalesce into either a hole or an annular ring upon annealing [17]. It is possible that the beam conditions may still be optimized in order to improve the probability that exposure 


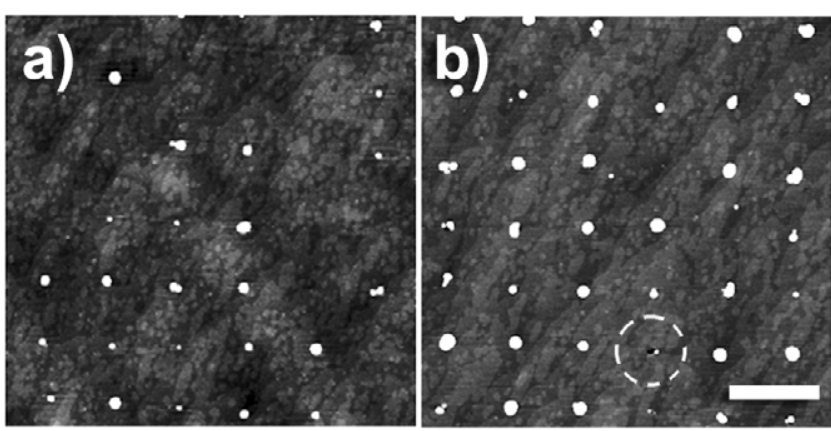

Figure 4. AFM images of a 2.0 ML InAs film deposited at $T=530{ }^{\circ} \mathrm{C}$ on GaAs upon hole arrays patterned $250 \mathrm{~nm}$ apart and at dwell times of (a) $560 \mu \mathrm{s}$ and (b) $1500 \mu \mathrm{s}$. The scale bar is $500 \mathrm{~nm}$, and the height scale is $10 \mathrm{~nm}$ in (a) and (b). The dotted circle in (b) indicates the presence of a quantum dot next to the irradiated hole it nucleated from.

to the ion beam will produce the desired feature with $100 \%$ fidelity at lower dwell times. The size of the holes increases with increasing dose. The open symbols in figure 3(a) show that the diameter of the holes increases from $30 \pm 10$ to $40 \pm 18 \mathrm{~nm}$ as the dwell time changes from 400 to $1600 \mu \mathrm{s}$. The depth increases from $1.5 \pm 1$ to $4 \pm 2 \mathrm{~nm}$ over the same range in ion dose (open symbols in figure 3(b)). However, it should be noted that when the holes become very narrow and deep, AFM is limited in its ability to accurately measure their true depth.

Growth of InAs upon these patterns results in the formation of regular arrays of quantum dots with a separation dictated by the dimensions of the pattern. Figures 4(a) and (b) show AFM micrographs of a 2.0 ML InAs film deposited at $T=530^{\circ} \mathrm{C}$ upon hole arrays, patterned using various dwell times, $560 \mu$ s (figure 4(a)) and $1500 \mu$ s (figure 4(b)). In general, dots nucleate on the sites where the holes were patterned, and the probability of having a single quantum dot at the expected location increases with longer dwell time, as shown in figure 2. In some instances, the hole is still visible next to a quantum dot (circled in figure 4(b)). The probability of the formation of a single dot is lower than that of a hole for any given dwell time, but roughly follows the same slope. There is also a non-zero probability of finding more than one dot at the intended location, which increases somewhat with increasing dwell time. The number of dots increases with dwell time. A dwell time of $800 \mu \mathrm{s}$ yields an average of $1.9 \pm 0.9$ dots, and a dwell time of $2000 \mu$ s yields an average of $5.8 \pm 1.2$ dots, which were tabulated from AFM images of one sample of $1.8 \mathrm{ML}$ InAs grown at $510^{\circ} \mathrm{C}$ on a FIB-irradiated surface with holes spaced $560 \mathrm{~nm}$ apart. For any given dwell time, the average quantum dot diameter is somewhat larger $(64 \pm 29 \mathrm{~nm}$ at $800 \mu \mathrm{s})$ than the average hole diameter $(42 \pm 13 \mathrm{~nm}$ at $800 \mu$ s), and weakly increases with increasing ion dose (closed symbols in figure 3 ).

The ratio of the height to the diameter of the FIBinduced quantum dots is 0.25 regardless of the dwell time. This compares favorably to reported aspect ratios of 0.24 and absolute sizes of quantum dots self-assembled without patterning [18]. This data shows that the ion irradiation or

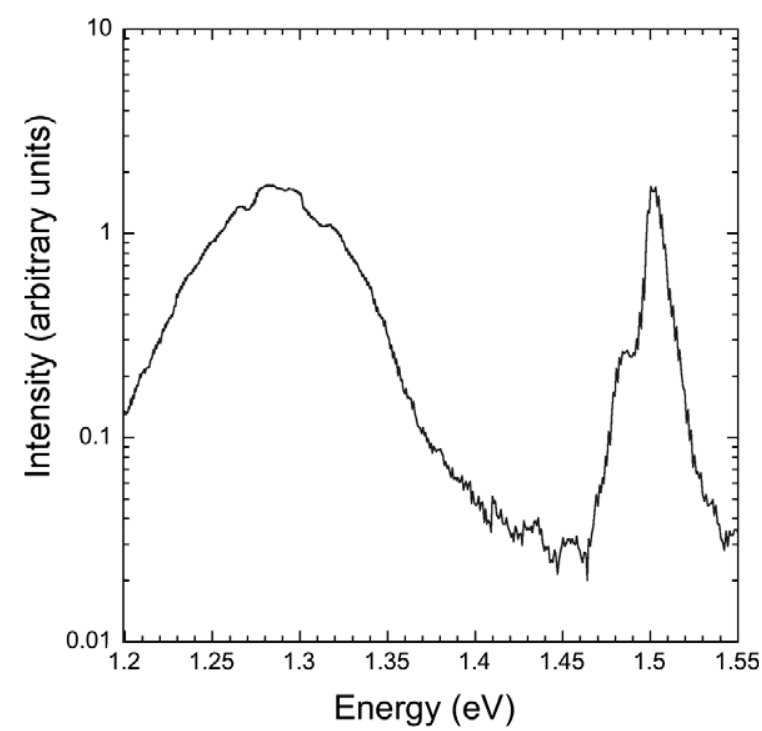

Figure 5. Photoluminescence spectra of FIB-modified surface. PL was performed at $77 \mathrm{~K}$ at a power of $15 \mathrm{~mW}$.

presence of any residual $\mathrm{Ga}^{+}$from the ion beam has no effect on the surface energy of the system, contrary to observations of FIB-induced quantum dots in the SiGe system [19]. If the ion beam did alter the surface energy, due to deposition of excess Ga from the beam for instance, then the aspect ratio of the FIBinduced islands would be expected to be different than selfassembled islands.

Samples for micro-photoluminescence (PL) were also grown to examine the optical activity of the FIB-directed quantum dots. These samples were grown similar to those described above, except a 10 period $2.5 \mathrm{~nm} \mathrm{GaAs} / 2.5 \mathrm{~nm} \mathrm{AlAs}$ superlattice was deposited prior to the quantum dot layer in order to amplify the PL emission, followed by a $10 \mathrm{~nm}$ GaAs spacer layer that was patterned by the FIB. The samples were then placed back into the chamber, and 1.8 ML InAs was deposited at $T=510^{\circ} \mathrm{C}$ and capped with $10 \mathrm{~nm}$ GaAs. PL measurements were made $e x$ situ with the sample mounted in a continuous-flow cryostat cooled to $77 \mathrm{~K}$ with liquid nitrogen. A $532 \mathrm{~nm}$ continuous-wave pump laser was focused with a $50 \times$ objective lens onto the FIB-irradiated regions. A CCD camera verified the laser beam, which had an estimated spot size of $2.6 \mu \mathrm{m}$, interrogated the irradiated regions. The PL signal was collected by the same objective lens, analyzed by a $50 \mathrm{~cm}$ monochromator, and detected with a liquid nitrogencooled InGaAs detector. An optical filter was placed at the entrance slit of the monochromator to block any backscattered pump light. Figure 5 shows that for an excitation power of $15 \mathrm{~mW}$, there is quantum dot emission at $1.28 \mathrm{eV}$ and emission from the substrate and cladding layers at $1.5 \mathrm{eV}$. These results are similar to those found for quantum dots assembled without patterning. For example, regular self-assembled InAs quantum dots had a PL emission at $1.21 \mathrm{eV}$ at $2 \mathrm{~K}$ [20] and $7 \mathrm{~K}$ [21], and at $1.3 \mathrm{eV}$ for $80 \mathrm{~K}$ [22]. Mehta et al report similar PL emission for FIB-induced quantum dots, with quantum dot emission at $1.18 \mathrm{eV}$ at $3 \mathrm{~mW}$ [23]. From these data we conclude that FIB irradiation does not hinder the PL emission 


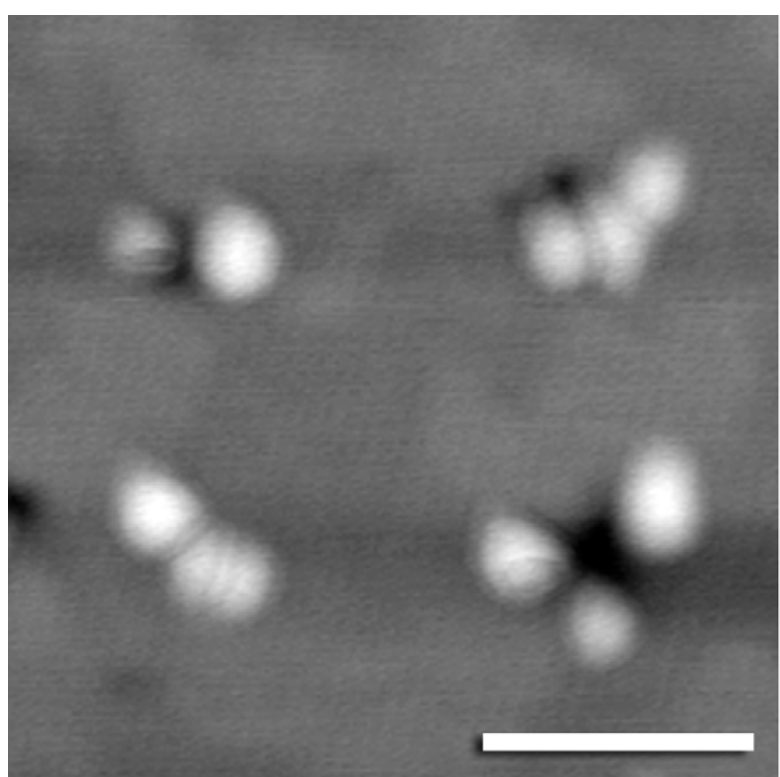

Figure 6. AFM image of a 1.8 ML-thick InAs film upon a pattern of holes spaced $140 \mathrm{~nm}$ apart and exposed to the beam $1600 \mu$ s per spot. The scale bar is $100 \mathrm{~nm}$ and the height scale is $15 \mathrm{~nm}$.

of InAs quantum dots. The width of the peak is likely due to the wide size distribution observed in these samples, which we hope to control by optimizing the patterning process.

It is not surprising that these FIB-induced holes act as nucleation sites in light of the fact that quantum dot formation has been observed on mesa tops [21] and at step edges [24]. In these experiments, quantum dots are not observed away from the pattern until a thickness of $2.2 \mathrm{ML}$, indicating that the pattern lowers the critical thickness for quantum dot formation [11]. In the case of the FIB-directed assembly observed in these experiments, it is not uncommon for two or three quantum dots to nucleate in the vicinity of a single hole, as seen in the AFM images of figures 4(a) and (b), and the plot in figure 2. The number of locations where at least one quantum dots has formed is consistently less than the number of holes observed for any given dwell time for these growth conditions, suggesting that either some holes are annihilated during the growth of the film, or the AFM is limited in its detection of small dots within the holes. As with the hole arrays, there is no observable effect of the periodicity of the array on the probability of finding one or more dots at a given location for these growth conditions.

The number of quantum dots per hole can be manipulated by altering the growth conditions, namely lowering the growth temperature. Figure 6 shows a high magnification AFM image that shows the details of multiple dot nucleation. In this image, the film is 1.8 ML-thick InAs deposited upon a pattern of holes spaced $140 \mathrm{~nm}$ apart with a beam exposure of $1600 \mu \mathrm{s}$ per spot and a growth temperature of $510^{\circ} \mathrm{C}$. In this lower temperature sample, many smaller dots nucleate at each hole. This is not unexpected, as the nucleation rate is higher at lower temperatures. Furthermore, the lower temperature film is thinner further leading to many small quantum dots.

In order to investigate the nucleation of quantum dots in response to patterned arrays of holes, we employ a kinetic Monte Carlo (KMC) model of crystal growth that take elastic relaxation of the lattice into account. We use a simple solidon-solid model [25-28] and assume that the migrating surface adatoms are units of GaAs or InAs. In other words, we do not take the effect of As overpressure into account. In this model only surface atoms can move and the hopping rate associated with the pth surface atom is denoted as $r_{\mathrm{p}}$. The hopping rate is modeled as

$$
r_{\mathrm{p}}=r_{0} \exp \left[\left(-E_{\mathrm{d}}-E+\Delta W\right) / k_{\mathrm{B}} T\right]
$$

where $E_{\mathrm{d}}$ is the diffusion barrier for InAs on GaAs, $k_{\mathrm{B}} T$ is the thermal energy, $r_{0}$ is the attempt frequency, and $E$ is the composite bonding energy term given by:

$$
E=N_{\mathrm{GG}} \gamma_{\mathrm{GG}}+N_{\mathrm{IG}} \gamma_{\mathrm{IG}}+N_{\mathrm{II}} \gamma_{\mathrm{II}}-5 \gamma_{\mathrm{IG}},
$$

$N_{\mathrm{GG}}$ is the total number of GaAs-GaAs bonds between atom $\mathrm{p}$ and its nearest and next nearest neighbors and the bond energy is denoted as $\gamma_{\mathrm{GG}}$. The quantities $N_{\mathrm{IG}}, N_{\mathrm{II}}, \gamma_{\mathrm{IG}}$, and $\gamma_{\mathrm{II}}$ are defined in an analogous way. (Note: for an isolated InAs atom on a GaAs substrate $E=0$.) The elastic energy $W$ is given as:

$$
\Delta W=W(\text { with atom } \mathrm{p})-W(\text { without atom } \mathrm{p}) .
$$

In our simulations we use a ball and spring model with springs connecting nearest and next to nearest neighbors, with spring constants $k_{L}$ and $k_{D}$ respectively. $W$ is the total elastic energy and computed by summing the energy of each spring. The computation of $\Delta W$ is rather expensive; however one can use the good approximation $\Delta W=\frac{3}{2} w_{\mathrm{p}}$, where $w_{\mathrm{p}}$ is the
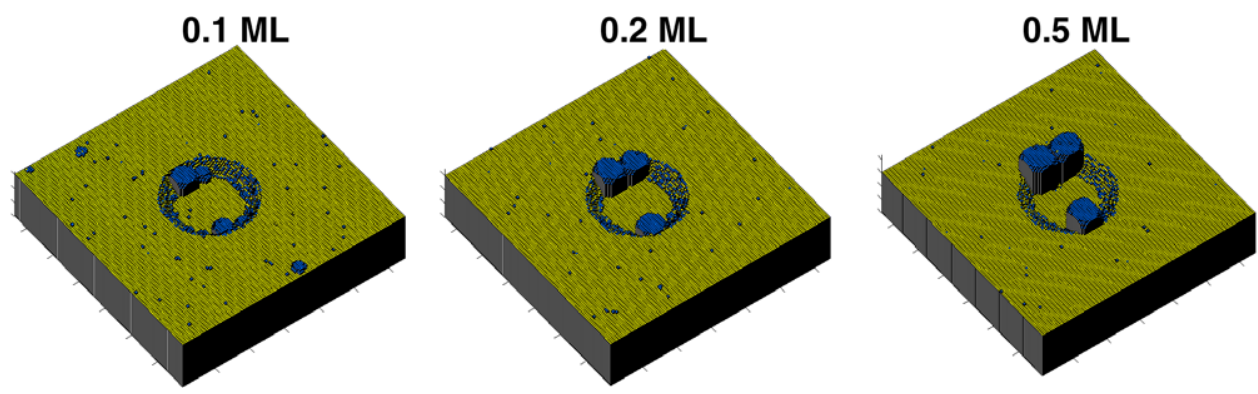

Figure 7. KMC simulation results as a function of deposition of InAs with the same growth parameters and starting morphology. The width of the computational cell is $128 \mathrm{au}$. 
total energy of the springs connected to atom $\mathrm{p}$ which is computationally faster. The system is then evolved using rejection-free KMC [29]. After each atom hop the elastic displacement field is updated. This is accomplished using the expanding box method combined with a Fourier multigrid method [28, 30-32].

In these calculations we take the strain of $7 \%$ of solidon-solid cubic crystal substrate (GaAs) and film (InAs), $r_{0}=$ $2 \times 10^{13} \mathrm{~s}^{-1}, E_{\mathrm{d}}=1 \mathrm{eV}, \gamma_{\mathrm{GG}}=\gamma_{\mathrm{IG}}=.18 \mathrm{eV}, \gamma_{\mathrm{II}}=.16 \mathrm{eV}$, $k_{L}=62 \mathrm{eV} / a^{2}$, and $k_{D}=30 \mathrm{eV} / a^{2}$. The values for $\gamma_{\mathrm{GG}}$, $\gamma_{\mathrm{IG}}, \gamma_{\mathrm{II}}, k_{L}$ and $k_{D}$ were chosen to match the bulk elastic properties of GaAs. These simulations were performed with a 128 atomic units $\times 128$ atomic units (au) matrix, and a height of $30 \mathrm{au}$. A half monolayer of atoms was deposited and allowed to interact with the buffer layer below, resulting in a threedimensional morphology.

The simulations qualitatively follow the experimental observations for quantum dot formation. Figure 7 presents the simulated morphology of the film growth as a function of deposited thickness. At the smallest thicknesses (0.1 ML) there are small nuclei on the inner perimeter, with a few small nuclei away from the hole. At higher deposited thickness, the nuclei away from the hole disappear and the nuclei within the perimeter of the hole grow and eventually protrude. It is difficult to determine whether quantum dot growth is accurately observed experimentally because probing the interior of holes of these size scales is hard to achieve with the AFM. The AFM tips have a radius of curvature of $8 \mathrm{~nm}$ and can only reliably image protruding dots. Experimentally, the number of locations where quantum dot formation is observed is consistently less than the number of holes observed for any given dwell time (figure 2). The simulations suggest that the holes at which no dots are observed are not necessarily annihilated during the growth of the film; rather, the quantum dots are completely within the holes and are thus undetectable by AFM.

The KMC simulations also replicate experimentally observed formation of quantum dots as a function of growth temperature. A minimum of four runs at different randomizations was used to achieve an average number of dots nucleated per hole. The simulation shows that an increase in the growth temperature from 750 to $850 \mathrm{~K}$ severely suppresses the nucleation rate of quantum dots around the patterned hole of $20 \mathrm{au}$ in diameter, from an average of $6.0 \pm 1.2$ dots to $1.6 \pm 0.6$ dots (figure 8(a)). This is in reasonable agreement with experimental results that show an average of $4.5 \pm 1.2$ dots at $T=510^{\circ} \mathrm{C}(783 \mathrm{~K})$ and $1.3 \pm 0.6$ dots at $T=530{ }^{\circ} \mathrm{C}$ $(803 \mathrm{~K}$ ) for a dwell time of $1600 \mu \mathrm{s}$. Temperature is also a reasonable predictor of whether a quantum dot nucleates away from the patterned hole. At $750 \mathrm{~K}$ in the simulations, an average of 2 dots nucleated away from the patterned area and for experimentally grown samples at growth temperatures below $510^{\circ} \mathrm{C}$, AFM reveals more nucleation of quantum dots away from the holes (not shown).

A large distribution in the initial hole diameters, shown as open symbols in figure 3(a), may contribute to the wide distribution in the quantum dot size (closed symbols in figure 3(a)) and multiple dot nucleation at a single hole site,
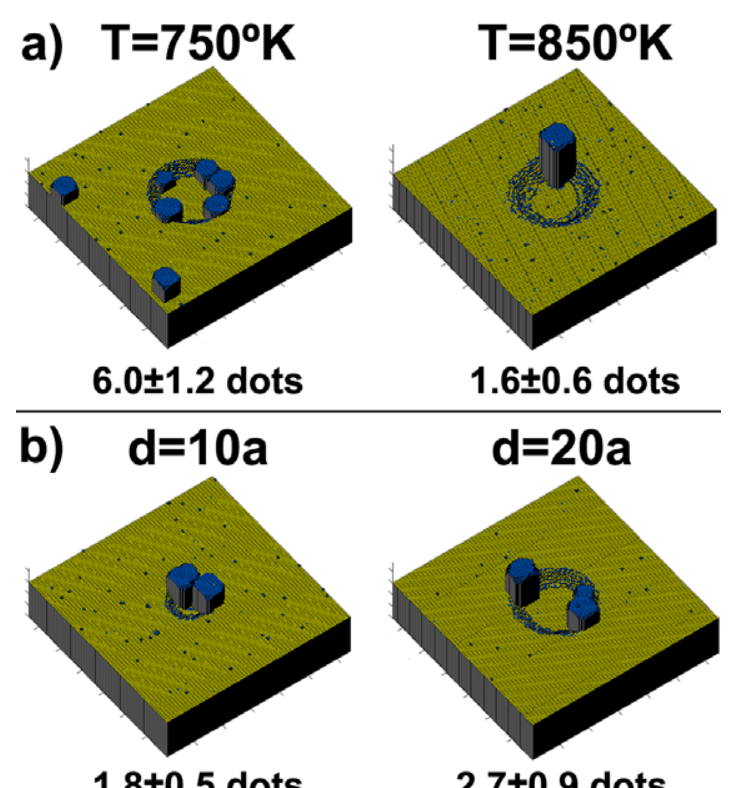

$1.6 \pm 0.6$ dots

$1.8 \pm 0.5$ dots

c) depth $=7 a$

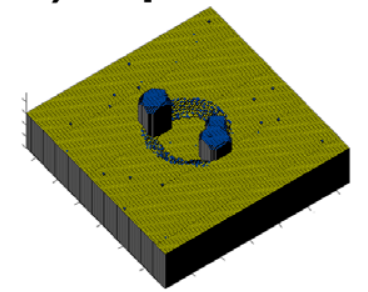

$2.7 \pm 0.9$ dots
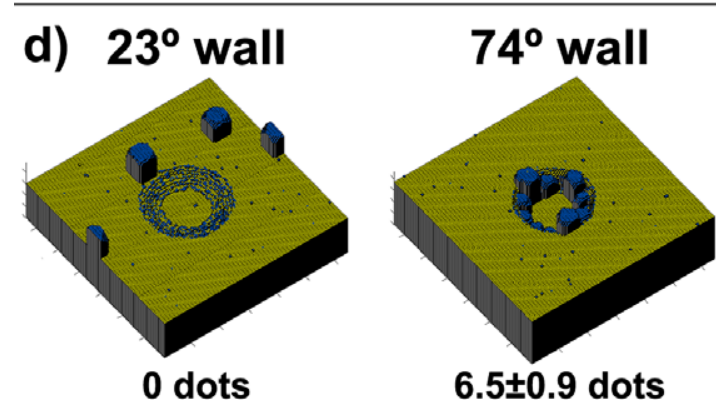

Figure 8. KMC simulation results for (a) varying temperature, (b) varying diameter, (c) varying depth, (d) varying wall slope (gradual $=23^{\circ}$, abrupt $=74^{\circ}$ with respect to the surface) of $0.5 \mathrm{ML}$ InAs deposited on GaAs. The number of dots is for an average of at least 4 different randomizations. The width of the computational cell is $128 \mathrm{au}$.

thus creating the wide PL peak observed in figure 5. From figure 3 it is clear that the diameter and depth change as a function of ion dose, but it is unclear which of these factors influence the number of quantum dots that form. Simulations give us the ability to change one parameter at a time to investigate these effects independently. When the hole diameter was doubled from 10 to 20 au as shown in figure 8(b), the number of quantum dots that formed increased only slightly, $2.1 \pm 0.7$ to $2.7 \pm 0.9$ dots, respectively. Instead, simulations show that the driving force of the increasing number of quantum dots per feature seems to be largely 
attributed to the depth and slope of the initial hole. Figure 8(c) shows that doubling the depth increases the number of dots nucleating per hole the dots from $2.7 \pm 0.9$ to $6.2 \pm 1.6$. Similarly, figure $8(\mathrm{~d})$ shows that when the slope of the hole wall is gradual, $27^{\circ}$ with respect to the surface, the dots only nucleate away from the hole. But when the slope is very sharp, $74^{\circ}$ with respect to the surface, there are many dots that form within the hole $(6.5 \pm 0.9)$. These simulations indicate that the depth and shape of the hole, and not necessarily the diameter, determines the number of quantum dots nucleated.

\section{Conclusions}

In this work FIB-induced morphologies created self-assembled quantum dots that were optically active. Variations of ion dose and other growth parameters, such as thickness, temperature, geometry of created holes, were examined both experimentally with AFM and also utilizing a KMC simulation with elastic contributions from the buffer layer. Size, fidelity, and the number of dots were strongly influenced by the FIB-irradiated dwell time. At low ion doses, holes are not always present, but increasing the ion dose increases the fidelity and also enlarges the holes. Multiple dots nucleate around a single hole when the temperature is decreased and when the hole surface area increases, i.e. when the hole diameter increases or depth increases.

\section{Acknowledgments}

This research was supported in part by a grant from Sandia National Labs (763642). Peter Smereka was supported, in part, by National Science Foundation grants DMS-0553487, DMS-0509124 and DMS-0810113. Thanks to Min W Kim and $\mathrm{P} \mathrm{C} \mathrm{Ku}$ for their assistance with the PL. And a big thank you to Aaron Dehne and Paul Rudzinski for their AFM analysis.

\section{References}

[1] Eberl K, Lipinski M O, Manz Y M, Winter W, Jin-Phillipp N Y and Schmidt O G 2001 11th Int. Winterschool on New Developments in Solid State Physics. Low-Dimensional Systems: Fundamentals and Applications (Feb. 2000); Physica E 9 164-74

[2] Livshits D A, Kovsh A R, Maleev N A, Zhukov A E, Ustinov V M, Ledentsov N N, Alferov Z I, Bimberg D, Lin G and Chi J 2003 Quantum Sensing: Evolution and Revolution from Past to Future (Jan. 2003); Proc. SPIE $4999524-30$

[3] Alloing B, Zinoni C, Zwiller V, Li L H, Monat C, Gobet M, Buchs G, Fiore A, Pelucchi E and Kapon E 2005 Appl. Phys. Lett. 86101908

[4] Ward M B, Karimov O Z, Unitt D C, Yuan Z L, See P, Gevaux D G, Shields A J, Atkinson P and Ritchie D A 2005 Appl. Phys. Lett. 86201111
[5] Nakamura Y, Schmidt O G, Jin-Phillipp N Y, Kiravittaya S, Muller C, Eberl K, Grabeldinger H and Schweizer H 2002 J. Cryst. Growth 242 339-44

[6] Watanabe S, Pelucchi E, Dwir B, Baier M, Leifer K and Kapon E 2004 11th Int. Conf. on Modulated Semiconductor Structures (July 2003); Physica E 21 193-8

[7] Kiravittaya S, Rastelli A and Schmidt O G 2005 Appl. Phys. Lett. 87243112

[8] Song H Z, Nakata Y, Okada Y, Miyazawa T, Ohshima T, Takatsu M, Kawabe M and Yokoyama N 2004 11th Int. Conf. on Modulated Semiconductor Structures (July 2003); Physica E 21 625-30

[9] Vandervelde T E, Atha S, Hull R, Pernell T L and Bean J C 2006 J. Vac. Sci. Technol. A 24 375-81

[10] McKay H A, Dehne A, Lee J Y and Millunchick J M 2007 Appl. Phys. Lett. 90163109

[11] McKay H, Rudzinski P, Dehne A and Millunchick J M 2007 Nanotechnology 18455303

[12] Kim Y K, Danner A J, Raftery J J and Choquette K D 2005 IEEE J. Sel. Top. Quantum Electron. 11 1292-8

[13] Kitslaar P, Strassner M, Sagnes I, Bourhis E, Lafosse X, Ulysse C, David C, Jede R, Bruchhaus L and Gierak J 2006 Microelectron. Eng. 83 811-4

[14] Menzel R, Bachmann A T and Wesch W 1999 Nucl. Instrum. Methods Phys. Res. B 148450

[15] Lugstein A, Basnar B and Bertagnolli E 2002 J. Vac. Sci. Technol. B $202238-42$

[16] Basnar B, Lugstein A, Wanzenboeck H, Langfischer H, Bertagnolli E and Gornik E 2003 J. Vac. Sci. Technol. B 21 927-30

[17] Portavoce A, Kammler M, Hull R, Reuter M C and Ross F M 2006 Nanotechnology 17 4451-5

[18] Yamaguchi K, Kaizu T, Yujobo K and Saito Y 2002 J. Cryst. Growth 237-239 1301-6

[19] Portavoce A, Hull R, Reuter M C and Ross F M 2007 Phys. Rev. B 76235301

[20] Henini M, Sanguinetti S, Brusaferri L, Grilli E, Guzzi M, Upward M D, Moriarty P and Beton P H 1997 Microelectron. J. 28 933-8

[21] Heitz R, Ramachandran T R, Kalburge A, Xie Q, Mukhametzhanov I, Chen P and Madhukar A 1997 Phys. Rev. Lett. 78 4071-4

[22] Tarasov G G, Mazur Y I, Zhuchenko Z Y, Maassdorf A, Nickel D, Tomm J W, Kissel H, Walther C and Masselink W T 2000 J. Appl. Phys. 88 7162-70

[23] Mehta M, Reuter D, Melnikov A, Wieck A D and Remhof A 2007 Appl. Phys. Lett. 91123108

[24] Placidi E, Arciprete F, Sessi V, Fanfoni M, Patella F and Balzarotti A 2005 Appl. Phys. Lett. 86241913

[25] Orr B G, Kessler D A, Snyder C W and Sander L 1992 Europhys. Lett. 1933

[26] Lam C H, Lee C K and Sander L M 2002 Phys. Rev. Lett. 8916102

[27] Lung M T, Lam C H and Sander L M 2005 Phys. Rev. Lett. 95086102

[28] Baskaran A, Devita J P and Smereka P 2009 Kinetic Monte Carlo simulation of strained heteroepitaxial growth with intermixing Contin. Mech. Thermodyn. submitted

[29] Blue J L, Beichl I and Sullivan F 1995 Phys. Rev. E 51 R867-8

[30] Russo G and Smereka P 2006 J. Comput. Phys. 214809

[31] Russo G and Smereka P 2006 Multisc. Model. Simul. 5130

[32] Schulze T P and Smereka P 2009 J. Mech. Phys. Solids 57 521-38 